\title{
ARIA masterclass 2018: From guidelines to real-life implementation*
}

Peter W. Hellings', Sven F. Seys², Gert Marien², loana Agache', Walter Canonica ${ }^{4}$, Philippe Gevaert ${ }^{5}$, Tari Haahtela ${ }^{6}$, Ludger Klimek, Joaquim Mullol ${ }^{8}$, Oliver Pfaar ${ }^{9}$, Glenis Scadding ${ }^{10}$, Guy Scadding ${ }^{11}$, Arunas Valiulis $^{12}$, ARIA masterclass discussants ${ }^{13}$, Jean Bousquet ${ }^{14}$, Benoit Pugin ${ }^{2}$
Rhinology 57: 0, $000-000,2019$ https://doi.org/10.4193/Rhin19.011

*Received for publication:

January 15, 2019

Accepted: May 9, 2019

${ }^{1}$ Allergy and Clinical Immunology Research Group, Department of Microbiology, Immunology \& Transplantation, KU Leuven, Leuven, Belgium ${ }^{2}$ European Forum for Research and Education in Allergy and Airway Diseases (EUFOREA), Brussels, Belgium and Allergy and Clinical Immunology Research Group, Department of Microbiology, Immunology \& Transplantation, KU Leuven, Leuven, Belgium

${ }^{3}$ Department of Allergy \& Clinical Immunology, University of Transylvania, Brasov, Romania

${ }^{4}$ Personalized Medicine, Asthma \& Allergy - Humanitas Clinical and Research Center, IRCCS - Rozzano (MI), Italy

${ }^{5}$ Upper Airways Research Laboratory, Department of Otorhinolaryngology-Head and Neck Surgery, University of Ghent, Belgium

${ }^{6}$ Skin and Allergy Hospital, Helsinki University Hospital, University of Helsinki, Helsinki, Finland

${ }^{7}$ Center for Rhinology and Allergology, Wiesbaden, Germany

${ }^{8}$ Rhinology Unit \& Smell Clinic, ENT Department, Hospital Clinic, IDIBAPS, Universitat de Barcelona, CIBERES, Barcelona, Spain

${ }^{9}$ Department of Otorhinolaryngolgy, Head and Neck Surgery, Section of Rhinology and Allergy, University Hospital Marburg, Philipps-Universitat Marburg, Germany

${ }^{10}$ Department of Allergy and Rhinology, Royal National Throat, Nose and Ear Hospital, University College London School of Medicine, London, UK

${ }^{11}$ Royal Brompton and Harefield NHS Trust, London, UK

12 Professor of Clinic of Children's Diseases of Institute of Clinical Medicine of Vilnius University Faculty of Medicine, Vilnius, Lithuania

${ }^{13}$ Listed in "Acknowledgements"

${ }^{14}$ University Hospital, Montpellier, MACVIA-France, Fondation partenariale FMC VIA-LR, Montpellier, France

\section{Abstract}

Over the past 20 years, ARIA (Allergic Rhinitis and its Impact on Asthma) has developed various guidelines for the treatment of allergic rhinitis (AR) and asthma multimorbidity. Over time, the ARIA initiative has evolved to ensure the highest level of bestpractices adoption in real life settings. It has evolved towards Integrated Care Pathways (ICPs) using mobile technology, and has now entered a new phase in which change management is key to provide an active and healthy life to all AR patients. With that in mind, the first ARIA masterclass was held on 12th September 2018 in Brussels, Belgium. The masterclass aimed at informing clinicians about the principles of change management, providing unbiased education on diagnosis and treatments, sharing the most recent research data on AR and multimorbidities, and creating a snowball effect to increase the adoption of best practices around the globe.

This report provides an overview of the ARIA masterclass concept, summarizes the key lectures and discussions, and gives an outline of the future key development.

Key words: ARIA, allergic rhinitis, asthma, masterclass 


\section{Introduction}

Respiratory allergies are on the rise and represent a serious health problem worldwide ${ }^{(1,2)}$. It is estimated that $20-30 \%$ of the European population suffers from allergic rhinitis (AR) ${ }^{(3)}$, with citizens from all countries, ethnic groups, and ages affected by the disease. AR influences social life, sleep, school and work productivity ${ }^{(4,5)}$, and in turn generate substantial indirect costs for the society ${ }^{(1)}$.

To find solutions for this ever-growing epidemic, an ARIA (AIlergic Rhinitis and its Impact on Asthma) -WHO (World Health Organization) workshop was organized for the first time in 1999, with the goal of better characterizing this allergic disease and proposing a stepwise action plan to address this socio-economic problem. Since that first meeting, the ARIA expert group has achieved numerous milestones contributing to a better management of AR and multimorbidities, including (but not limited to):

- Novel classification of AR by symptom's duration (intermittent, persistent) and disease severity (mild, moderate/ severe) ${ }^{(6)}$

- Official recognition of the significance of multimorbidities in $A R^{(6)}$.

- $\quad$ Release of the first exhaustive ARIA guidelines (2008 \& 2010), including an overview of evidence-based diagnostic methods, treatments, and care management ${ }^{(7,8)}$.

- Development of a simple, guideline-based, and patientcentered mobile app. (i.e. MASK Allergy Diary) to help answering some of the unmet needs in AR and asthma ${ }^{(9)}$.

- Creation of multi-disciplinary Integrated Care Pathway (ICP) for AR and multimorbidities (10).

- Merge with the non-profit organization EUFOREA to increase the outreach and impact of ARIA activities (www. euforea.eu).

- Proposal for a Change Management strategy to reach a worldwide reduction of $A R$ and asthma burden ${ }^{(11)}$

\section{Incentive for the creation of the ARIA masterclass and key objectives}

In spite of the various guidelines, pocket guides, and other digital tools developed through the ARIA initiative, many clinicians remain unexposed to this evidence-based information, or some remain uncertain of the advantages and drawbacks of the many AR treatment options available. Thus, various allergists part of the ARIA expert group have observed suboptimal management of patients around the globe, resulting in poorly controlled $A R^{(12)}$ and additional cost due to improper medications ${ }^{(13)}$. Moreover, real-life evidence shows that most AR patients do not follow guidelines and have a very poor adherence to treatment (14-16)

Therefore, the ARIA masterclass was created to fill this gap and inform a large number of ambassadors, i.e. clinicians, which will further spread the best practices at national and regional levels. The first ARIA masterclass took place on the 12th September 2018 in Brussels, Belgium, and was attended by over 200 clinicians from 21 countries (Figure 1). An educational program was designed to update the participants on the latest data from clinical trials as well as recent real-life evidences. In addition, clinicians received practical information about the best practices, including the latest diagnostic tools for optimal patients' stratification, and a presentation of all therapeutic strategies available nowadays for AR and multimorbidity. Finally, the role and benefits of novel technologies (mHealth tools) were presented. The lectures were fully recorded and are available on the EUFOREA website (www.euforea.eu).

\section{Barriers and strategies to improve the effectiveness of care management in AR}

Challenge of AR and multimorbidities

Multimorbidity has been defined as the presence of one or more additional disorders co-occurring with a primary disease, or the effect of such additional disorders ${ }^{(17)}$. AR may be either associated with asthma (multimorbidity) or as a single disease, and these two phenotypes differ widely ${ }^{(18-20)}$. Data provided by several birth cohorts show the trajectories of patients with allergic symptoms ${ }^{(21)}$ and a presence of allergic multi-morbidity ( 2 or more of asthma, rhinitis and eczema) occurring in over $10 \%$ of young adults. However, multimorbidity was not associated with lgE sensitization ${ }^{(22)}$. The vast majority of asthmatics have rhinitis, and AR is a risk factor for new onset asthma as well as a risk factor for asthma in the next generation ${ }^{(23)}$. Treating AR may therefore improve asthma control although a definite conclusion cannot be reached with current data. AR was also associated with several other conditions: AR is strongly associated with conjunctivitis ${ }^{(24,25)}$. Although not clearly demonstrated to be a risk factor for chronic rhinosinusitis, IgE sensitization appears to be a risk factor for both upper and lower respiratory tract infections ${ }^{(26)}$. AR may be associated with otitis media with effusion in children ${ }^{(27)}$. Finally, pollen-food syndrome is common in European children and adults with AR and birch pollen sensitization ${ }^{(17)}$

The need for better symptom control

The cardinal symptoms in AR are runny or blocked nose, sneezing and itchy nose or eyes. These can be extremely troublesome, and the consequences of such symptoms cannot be neglected, with impaired sleep quality perhaps being the most vital (experienced by $50.3 \%$ of adults and $37.3 \%$ of children with AR) ${ }^{(28-31)}$. Consequent tiredness and poor concentration impinges on quality of life, on work and school performance and productivity (4). Unproductivity for $2.3 \mathrm{~h}$ per working day due to AR symptoms costs $\$ 593$ per person per year ${ }^{(32)}$. This is greater than that for heart disease, asthma, diabetes, hypertension and respiratory 


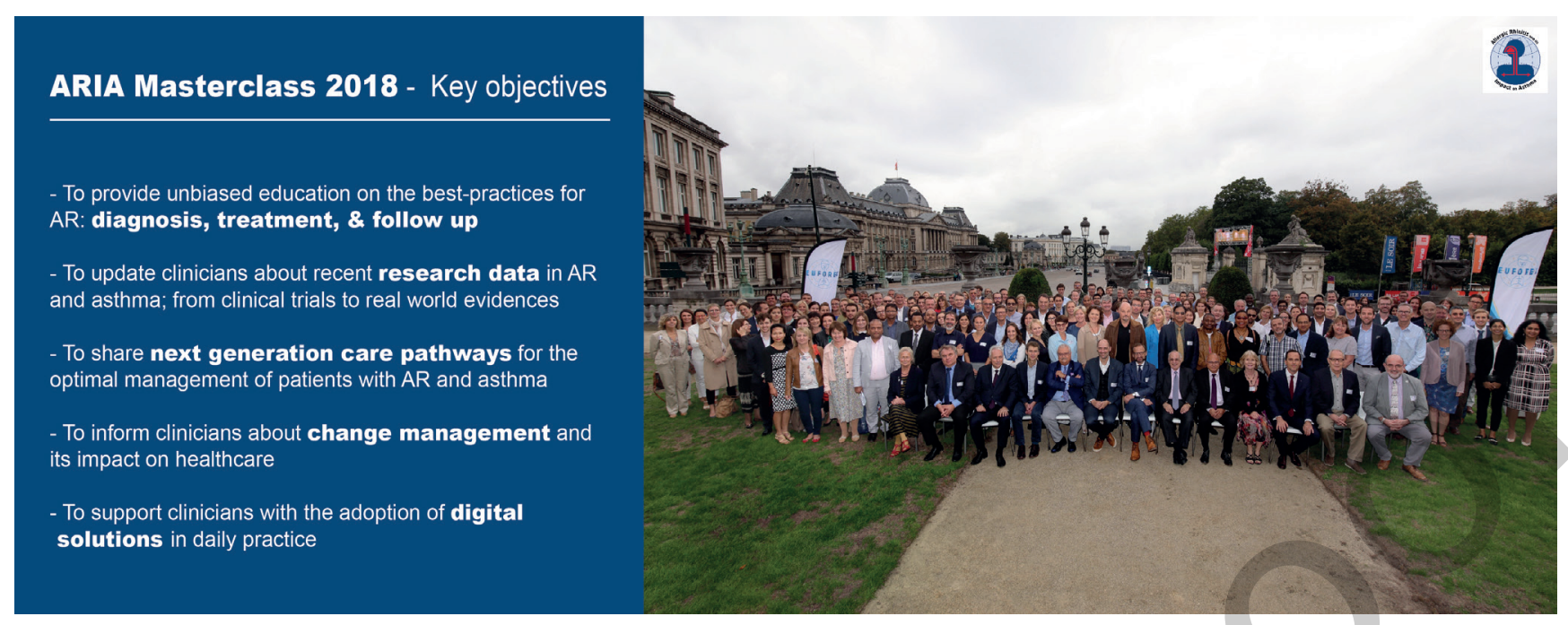

Figure 1. Impression of the ARIA Masterclass 2018 and key objectives.

illnesses combined ${ }^{(32)}$. Uncontrolled AR carries a high social burden in other respects: it is socially embarrassing to be seen sneezing, sniffing, or blowing the nose. Loss of smell has been also associated to severe and uncontrolled AR, both in adult (33) and children ${ }^{(34)}$. AR is associated with psychological disturbance and is a risk factor for depressive mood in pre-adolescents ${ }^{(35)}$. Practical issues exist: untreated AR can impair driving ability ${ }^{(36)}$, and this is worsened if inappropriate treatment such as sedating antihistamines are used ${ }^{(37)}$.

Currently many AR sufferers report poor symptom control and are willing to pay for a treatment for allergic rhinitis which works quickly and provides complete symptom relief ${ }^{(38,39)}$. Additionally, allergen immunotherapy (AIT) holds curative potential for allergic diseases and therefore represents an important treatment strategy to tackle the global rise of allergies ${ }^{\left({ }^{40,41)} \text {. The right }\right.}$ combination of both AIT \& pharmacotherapy holds the potential to i) control patients' symptoms, and ii) change the course of the allergic disease ${ }^{(40-44)}$. Another important issue is the need for continuous or on-demand treatment both in asthma ${ }^{(45,46)}$ or in rhinitis. In that direction, ICP provide a multi-stakeholder approach to the management of AR with pharmacists ${ }^{(47)}$ and GPs ${ }^{(48)}$.

Patient participation and shared-decision making In the digital era, where all information is readily accessible on the smart phone, we are moving from the "guideline era" to a "patient partnership era" for change management ${ }^{(11)}$. We also call it shared-decision making, participatory medicine, health consumerism, or patient-centered care, which traduces an experience of transparency, individualization, recognition, respect, dignity, and choice in all matters in health care ${ }^{(49)}$. Less dictating about compliance and adherence, and more collaborative with the patient. mHealth is an important solution to meet the growing demand for care. Mobile applications increase health literacy, bridge patient-physician communication, and increase patient participation. The problems now reside in the lack of research and testing the application before going live as well as patient willingness to use the apps over time ${ }^{(50,51)}$. Personal counselling of the patient by a professional is still critical. One important example is guided self-management, which dramatically reduced asthma exacerbations during the Finnish asthma (1994-2004) and allergy (2008-2018) programmes ${ }^{(52,53)}$. Note that in paediatric care, such mutual partnership is often more complicated, because legal and ethical issues regarding children are barely defined. In addition, children of different ages have different levels of competence regarding self-care and health. Since chronic diseases span over different stages of a child's development, shared-decision making must be constantly re-evaluated to the changing levels of autonomy and needs of a growing child. Nonetheless, parents or caregivers should be included in the process of shared-decision making. Altogether, in the modern health-care, patients have now more rights but also more responsibility of their own health.

Next-generation care pathways in AR and multimorbidities The transformation of the health care system for integrated care needs support via organizational health literacy. ARIA Phase 4 proposes a change management strategy to increase selfmedication and shared decision making in rhinitis and asthma multimorbidity ${ }^{(11)}$. MASK (Mobile Airways Sentinel Network) is a new development of the ARIA initiative ${ }^{(14,15,24)}$. It has developed and validated IT evidence-based tools. These tools can inform patient decisions on the basis of a self-care plan proposed by health care professionals. In collaboration with professional and patient organizations, POLLAR (Impact of Air Pollution on Asthma and Rhinitis, EIT Health) ${ }^{(54)}$ is proposing real-life care pathways which are centred around the patient and use 
mHealth monitoring of environmental exposure. Patient participation, health literacy and self-care are all included through technology-assisted "patient activation", implementation of care pathways by pharmacists and next-generation guidelines assessing the recommendations of GRADE guidelines using real-world evidence (RWE). The EU political agenda is of major importance in supporting healthcare transformation ${ }^{(55)}$ and MASK has been recognized by DG Santé as a Good Practice in the field of digitally-enabled, integrated, person-centred care.

\section{The value of Real World Evidence}

Because AR is often associated with various multimorbidities, randomized controlled trials (RCTs) cannot cover all forms of disease nor all phenotypes of patients ${ }^{(56)}$. RCTs are, by their nature, designed to answer narrowly defined scientific questions while controlling as many variables as possible ${ }^{(57-59)}$. While necessary for drug registration, this approach offers little insight into the acceptability and practicality of implementing new interventions into clinical practice. RWE should therefore complement data already gathered from well-designed RCTs. However, in order to be considered, such real-life evidence needs to be both published and of sufficiently high quality. For example, in the case of AIT, RCTs are not suitable for rare allergens, since not enough patients may exist in individual indications ${ }^{(60)}$, they may not provide sufficient safety data ${ }^{(61,62)}$ or may not provide pharmacoeconomic data ${ }^{(63,64)}$. Real-life studies fundamentally differ from RCT studies because they do not influence or change the normal patient-physician interaction in any way and incorporate the variability of everyday life, yielding results that are generalizable to a much broader patient population ${ }^{(65,66)}$. The variability intrinsic to this type of study means they are limited in establishing a direct cause and effect relationship. However, bias can be minimized by identification of confounding factors and use of rigorous analytical methods ${ }^{(57)}$. Validated outcome measures such as Visual Analogue Scales (VAS) have been applied to reallife studies ${ }^{(67,68)}$ and mobile health technologies like MASK are broadly available ${ }^{(15,69)}$.

\section{Conclusion}

A recent short study revealed that allergic allergy specialists behave like patients ${ }^{(70)}$, showing a real disconnection between physician's prescription and patient's behavior for the treatment AR. Indeed, the majority of allergists prescribe medications for the entire pollen season, but the vast majority of patients use their medications on-demand when their symptoms are not well controlled ${ }^{(15)}$. The goal of the ARIA masterclass was therefore to intend a reconnection between physicians and patients, by promoting a patient-centered approach, with a therapeutic strategy tailored to the patient needs but also to their preferences. The use of ICPs and mHealth can facilitate this change in care management. In turn, this would results in better symptoms controls for patients, and a substantial reduction of indirect cost for the society.

\section{Acknowledgements}

ARIA discussants: Nada Al-Muhandis ${ }^{1}$, Sarah Allatt ${ }^{2}$, Robert Almeyda ${ }^{3}$, Patricia Annand ${ }^{4}$, Marina Atanaskovic Markovic ${ }^{5}$, leva Bajoriūniené ${ }^{6}$, Abhijit-Neil Banik ${ }^{7}$, Humera Barbar-Craig ${ }^{8}$, James Barraclough ${ }^{9}$, Cécile Bedfert ${ }^{10}$, Ana Bedrock ${ }^{11}$, Mieke Beel ${ }^{12}$, David Bonilla Parra ${ }^{13}$, Elena Bradatan ${ }^{14}$, Michael Bras ${ }^{15}$, Detlef Brehmer ${ }^{16}$, David Brinkman ${ }^{17}$, Marisela Cardier Suárez ${ }^{18}$, Marina Carrasco Llatas $^{19}$, Petar Chipev ${ }^{20}$, Angela Cooper ${ }^{21}$, Dennis Corbett ${ }^{22}$, Caroline $\mathrm{Cox}^{23}$, Tony $\mathrm{Cox}^{24}$, Melinda Décsi ${ }^{25}$, Christos Dantsis ${ }^{26}$, Danielle Davies $^{27}$, Belen de la Hoz ${ }^{28}$, Maria Teresa Dordal Culla ${ }^{29}$, Astrid Dworan-Timler ${ }^{30}$, Stefan Edlinger ${ }^{31}$, Mohamed El Rakhawy ${ }^{32}$, Tariq El-Shanawany ${ }^{33}$, Wassim Elhendi Halawa ${ }^{34}$, Jean-Luc Fauquert ${ }^{35}$, Aoife Folliard ${ }^{36}$, Karen Frankland ${ }^{37}$, James Gardner ${ }^{38}$, Julian Gaskin $^{39}$, Tamazoust Guiddur ${ }^{40}$, Kerry-Ann Hanks ${ }^{41}$, Brendan Hanna ${ }^{42}$, Benjamin Heinz ${ }^{43}$, Roland Hettige ${ }^{44}$, Alice Horisberger ${ }^{45}$, Maxime Hosotte $^{46}$, David Howe ${ }^{47}$, Valerie Hox ${ }^{48}$, Sonna Ifeacho ${ }^{49}$, Anurag Jain $^{50}$, Mario Jaramillo ${ }^{51}$, Juan Miguel Jimenez Morales ${ }^{52}$, Savita Kale $^{53}$, Yvonne Kamper ${ }^{54}$, Gaurav Kansal ${ }^{55}$, Lenka Kapustova ${ }^{56}$, Dheeraj Karamchandani ${ }^{57}$, Siew Keh ${ }^{58}$, Ute Kerr ${ }^{59}$, Maha Khan ${ }^{60}$, Mitja Košnik ${ }^{61}$, Matej Kolar ${ }^{62}$, Beate Koos ${ }^{63}$, Kosta Kostov ${ }^{64}$, Helga Kraxner $^{65}$, Violeta Kvedarienè ${ }^{66}$, Simon Lawton ${ }^{67}$, Sébastien Lefevre $^{68}$, Eirini Lentari ${ }^{69}$, Martin Lindenberger ${ }^{70}$, Ralph Litschel ${ }^{71}$, Miha Ložar ${ }^{72}$, Marta Lomikovska ${ }^{73}$, Clemens Luckhardt ${ }^{74}$, Júlia Lukits $^{75}$, Oksana Marchuk ${ }^{76}$, Caroline Marlair ${ }^{77}$, Katleen Martens ${ }^{78}$, Jose Maria Mateo ${ }^{79}$, Stephen Mc Donald ${ }^{80}$, María Isabel Menaches Guardiola ${ }^{81}$, Mohammad Mohammad ${ }^{82}$, Joana Moreno Cosme $^{83}$, Montio Morgan ${ }^{84}$, Abigail Morris ${ }^{85}$, Tihomir Mustakov ${ }^{86}$, Shahid Nadeem ${ }^{87}$, Attila Nagy ${ }^{88}$, Romina Nemecek ${ }^{89}$, Barbara Nemeth $^{90}$, Catherine Neukirch ${ }^{91}$, Ihab Odeh ${ }^{92}$, Shalini Patiar ${ }^{93}$, Milena Pavic ${ }^{94}$, Armony Peglion ${ }^{95}$, Julie Pentland ${ }^{96}$, Anne Christine Perrot de Corgnol ${ }^{97}$, Otilia Petrovicova ${ }^{98}$, Krizia Piccininni ${ }^{99}$, Valeryi Piont ${ }^{100}$, Todor Popov ${ }^{101}$, Emese Popovics ${ }^{102}$, Fabienne Portejoie ${ }^{103}$, Santiago Quirce ${ }^{104}$, Bhaskar Ram ${ }^{105}$, Agnè Ramonaitè ${ }^{106}$, Abdul Razzak Juratli ${ }^{107}$, Pilar Rico ${ }^{108}$, Carsten Ritterhoff ${ }^{109}$, Alasdair Robertson ${ }^{110}$, Rosa Rodrigues Fernandes ${ }^{111}$, Sonja Runge ${ }^{112}$, Dermot Ryan ${ }^{113}$, Rafael Sanchez Gutierrez ${ }^{114}$, Isabelle Schatteman ${ }^{115}$, Sophie Scheire ${ }^{116}$, Aleksandra Schuhmacher-Koval ${ }^{117}$, Esteban Scola Pliego ${ }^{118}$, Rishi Shukla ${ }^{119}$, Derrick Siau ${ }^{120}$, Maaike Siemers ${ }^{121}$, Brigita Sitkauskienè ${ }^{122}$, Tomas Slomskis ${ }^{123}$, Tanja Soklič Košak ${ }^{124}$, Kato Speleman ${ }^{125}$, Thomas Spindler ${ }^{126}$, Jos Straetmans ${ }^{127}$, Dina Striūpaitienè ${ }^{128}$, Werner Sunk ${ }^{129}$, Rajendra Tadikonda ${ }^{130}$, Muhammad Tariq ${ }^{131}$, Barbara Terporten ${ }^{132}$, Shamim Toma ${ }^{133}$, Mirabela Tor $^{134}$, Paraskevi Tsirevelou ${ }^{135}$, Matthias Urban ${ }^{136}$, Jure Urbančič ${ }^{137}$, Lisa van der Putten ${ }^{138}$, Vlad-Corneliu Vasiliu' ${ }^{139}$, Nataliia Vesilyk ${ }^{140}$, Anna-Sophie Vink ${ }^{141}$, Elena Vriess-Uss ${ }^{142}$, Kieuhanh Vu ${ }^{143}$, Svitlana Yaremchuk $^{144}$, Patrick Yong ${ }^{145}$, Tosief Zahoor ${ }^{146}$, Mihaela Zidarn ${ }^{147}$.

${ }^{1}$ Castle Hill Hospital, Hull, UK. ${ }^{2}$ Royal Manchester Children's 
Hospital, Manchester, UK. ${ }^{3}$ Royal Berkshire Hospital, Reading, UK. ${ }^{4}$ Queen Margaret Hospital, Dunfermiline, UK. ${ }^{5}$ University Children's Hospital Tirsova, Belgrade, Serbia. ${ }^{6} \mathrm{Hospital}$ of Lithuanina University of Health Science Kaunas Clinics, Kaunas, Lithuania. ${ }^{7}$ Park Farm Surgery, Derby, UK. ${ }^{8}$ East Surrey Hospital, Redhill, UK. ${ }^{9}$ New Cross Hospital, Wolverhampton, UK. ${ }^{10}$ Cabinet médical, Vannes, France. ${ }^{11} \mathrm{MACVIA-France,} \mathrm{Fondation} \mathrm{partenariale} \mathrm{FMC}$ VIA-LR, Montpellier, France. ${ }^{12}$ Zorgsaam Ziekenhuis, Terneuzen, Netherlands. ${ }^{13}$ Hospital Vall d’Hebrón, Barcelona, Spain. ${ }^{14}$ Belgian Association for Continuing Education in Allergology (ABFORCAL) , Brussels, Belgium. ${ }^{15}$ ALK Benelux, Almere, Netherlands. ${ }^{16}$ Praxis, Göttingen, Germany. ${ }^{17}$ ENT Dept, Beaumont Hospital, Dublin , Ireland. ${ }^{18} \mathrm{Complejo}$ Hospitalario de Navarra, Pamplona, Spain. ${ }^{19} \mathrm{Hopistal}$ Universitario Doctor Peset, Valencia, Spain. ${ }^{20}$ Military Medical Academy, Sofia, Bulgaria. ${ }^{21}$ Royal Stoke University Hospital, Stoke, UK. ${ }^{22}$ Royal Blackburn Hospital, Blackburn, UK. ${ }^{23}$ Jessa Hospital, Hasselet, Belgium. ${ }^{24}$ Jessa Hospital, Hasselet, Belgium. ${ }^{25}$ Borsod-Abaúj-Zemplén County Central Hospital, Miskolc, Hungary. ${ }^{26}$ HNO Praxis Laufer \& Dantsis, Wuppertal, Germany. ${ }^{27}$ University Hospital Aintree, Liverpool, UK. ${ }^{28} \mathrm{Hospital}$ Universitario Ramon y Cajal, Madrid, Spain. ${ }^{29} \mathrm{Hospital}$ Bellvitge, Barcelona, Spain. ${ }^{30}$ Allergiezentrum Neusiedl, Neusiedl, Austria. ${ }^{31}$ Mylan, Vienna, Austria. ${ }^{32} \mathrm{Al}$ Garhoud private Hospital, Dubai, Dubai. ${ }^{33}$ University Hospital of Wales, Cardiff, UK. ${ }^{34} \mathrm{Hospital}$ Punta de Europa, Cádiz, Spain. ${ }^{35}$ Centre Hospitalier Universitaire de Clermont-Ferrand, Clermont-Ferrand, France. ${ }^{36}$ Mayo General Hospital, Castlebar, Ireland. ${ }^{37}$ Freelance Primary Care Respiratory Nurse, Manchester, UK. ${ }^{38}$ Great North Children's Hospital, Newcastle, UK. ${ }^{39}$ Bristol Royal Hospital for Children, Bristol, UK. ${ }^{40}$ Invasive Bacterial Infections Unit, National and Reference Centre for Meningococci, Institut Pasteur, Paris, France. ${ }^{41}$ Great Western Hospital, Swindon, UK. ${ }^{42}$ Ulster Hospital, Ulster, UK. ${ }^{43}$ Praxis, Schaffhausen, Switzerland. ${ }^{44}$ Wexham Park Hospital, Slough, UK. ${ }^{45} \mathrm{CHUV}$, Lausanne, Switzerland. ${ }^{46}$ Mylan, Paris, France. ${ }^{47}$ University Hospital Coventry \& Warwickshire, Coventry, UK. ${ }^{48} \mathrm{Cliniques}$ Universitaires Saint-LuC . Division of Otorhinolaryngology, Brussels, Belgium. ${ }^{49}$ Whipps Cross Hospital, London, UK. ${ }^{50}$ Queen's Hospital, Romford, UK. ${ }^{51}$ West Wales General Hospital, Carmarthen, UK. ${ }^{52} \mathrm{Hospital}$ Universitario Nuestra Señora de Valme, Sevilla, Spain. ${ }^{53}$ Hereford County Hospital, Hereford, UK. ${ }^{54}$ Mylan, Vienna, Austria. ${ }^{55}$ Whipps Cross Hospital, London, UK. ${ }^{56} \mathrm{~K}$ linika detí a dorastu, Univerzitná nemocnica Martin, Martin, Slovakia. ${ }^{57}$ University Hospitals Coventry \& Warwickshire, Coventry, UK. ${ }^{58}$ Queen Elizabeth University Hospital, Glasgow, UK. ${ }^{59}$ Kantonsspital, St.Gallen, Switzerland. ${ }^{60}$ Manchester Foundation Trust, Manchester, UK. ${ }^{61}$ University Clinic of Respiratory and Allergic Diseases, Golnik, Slovenia. ${ }^{62}$ General Hospital Celje, Celje, Slovenia. ${ }^{63}$ Mylan, Bad Homburg, Germany. ${ }^{64}$ Military Medical Academy, Sofia, Bulgaria. ${ }^{65}$ Semmelweis University Department of Otorhinolaryngology, Head and Neck Surgery, Budapest, Hungary. ${ }^{66} \mathrm{Hospital}$ of Vilnius University Santaros Clinics, Vilnius,
Lithuania. ${ }^{67} \mathrm{ALK}$, Copenhagen, Denmark. ${ }^{68} \mathrm{CHR}$ Metz Nancy, Ars Laquenexy, France. ${ }^{69}$ Addenbrooke's Hospital, Cambridge, UK. ${ }^{70}$ Mylan, Bad Homburg, Germany. ${ }^{71} \mathrm{Hals}$ Nasen Ohren Praxis, St.Gallen, Switzerland. ${ }^{72}$ Mylan, Ljubljana, Slovenia. ${ }^{73}$ Department of clinical immunology and allergology, Lviv National Medical University, Lviv, Ukraine. ${ }^{74}$ Praxis, Augsburg-Pfersee, Germany. ${ }^{75}$ Szent László Hospital, Budapest, Hungary. ${ }^{76}$ Mylan, Kiev, Urkraine. ${ }^{77}$ Médecin ORL au CHU UCL Namur site Ste Elisabeth, Namur, Belgium. ${ }^{78} \mathrm{KU}$ Leuven, Leuven, Belgium. ${ }^{79}$ Mylan, Madrid, Spain. ${ }^{80}$ Royal United Hospital Bath, Bath, UK. ${ }^{81} \mathrm{Hospital}$ Universitario San Juan, Alicante, Spain. ${ }^{82}$ South Infirmary Victoria University Hospital , Cork , Ireland. ${ }^{83}$ Chuc, Coimbra, Portugal. ${ }^{84}$ Basildon \& Thurrock University Hospital, Basildon, UK. ${ }^{85}$ Mylan, Hatfield, UK. ${ }^{86}$ Clinic of Allergology and Clinical Immunology, Alexandrovska Hospital, Sofia, Bulgaria. ${ }^{87}$ Walsall Manor Hospital, Walsall, UK. ${ }^{88}$ Semmelweis University Clinic of Pulmonology, Budapest, Hungary. ${ }^{89}$ Militär-Medizinisches Zentrum Heeresspital Wien, Wien, Austria. ${ }^{90}$ Mylan, Komarom, Hungary. ${ }^{91}$ Pneumologie, Hôpital Bichat-Claude Bernard, Assistance PubliqueHôpitaux de Paris, Paris, France. ${ }^{92}$ Mylan, Dubai, Dubai. ${ }^{93}$ Princess Alexandra Hospital, Harlow, UK. ${ }^{94}$ General Hospital, Pancevo, Serbia. ${ }^{95}$ Mylan, Paris, France. ${ }^{96}$ Great North Children's Hospital, Newcastle, UK. ${ }^{97}$ Hôpital François Quesnay, Mantes la Jolie, France. ${ }^{98} \mathrm{~K}$ linika detí a dorastu, Univerzitná nemocnica Martin, Martin, Slovakia. ${ }^{9}$ University of Bari, Bari, Italy. ${ }^{100}$ Mylan, Live, Ukraine. ${ }^{101}$ Medical Center Excelsior, Sofia, Bulgaria. ${ }^{102}$ Mylan, Komarom, Hungary. ${ }^{103} \mathrm{MACVIA-France,} \mathrm{Fondation} \mathrm{partenariale} \mathrm{FMC}$ VIA-LR, Montpellier, France. ${ }^{104}$ ENT Department, Hospital Clínic, Madrid, Spain. ${ }^{105}$ Aberdeen Royal Infirmary, Aberdeen, UK. ${ }^{106}$ Republic Hospital of Klaipeda, Klaipeda, Lithuania. ${ }^{107} \mathrm{Al}$ Zahra Hospital, Dubai, Dubai. ${ }^{108}$ Respiratory Immunoallergy, IDIBAPS, Madrid, Spain. ${ }^{109}$ Praxis, Altenstadt, Germany. ${ }^{110}$ Royal Alexandra Hospital, Paisley, UK. ${ }^{111}$ Centro Hospitalar Lisboa Norte - SantaMaria, Lisboa, Portugal. ${ }^{112}$ Praxis, Neuenhagen, Germany. ${ }^{13}$ Uni of Edinburgh, Edinburgh, UK. ${ }^{114}$ Hospital Universitario Reina Sofia, Córdoba, Spain. ${ }^{115}$ ENT at European Institute for ORL, Sint Augustinus, Antwerp, Belgium. ${ }^{116}$ Universiteit Ghent, Ghent, Belgium. ${ }^{117}$ Praxis, Gensingen, Germany. ${ }^{118}$ Hospital Gregorio Marañon, Madrid, Spain. ${ }^{119}$ Great Western Hospital, Glasgow, UK. ${ }^{120}$ Macclesfield District General Hospital, Macclesfield, UK. ${ }^{121}$ ZGT, Almelo, the Netherlands. ${ }^{122} \mathrm{Hospital}$ of Lithuanina University of Health Science Kaunas Clinics, Kaunas, Lithuania. ${ }^{123} \mathrm{UAB}$ Kardiolitos klinikos (JSC Kardiolita Clinics), Vilnius, Lithuania. ${ }^{124}$ University Clinical Center, Ljubljana, Slovenia. ${ }^{125} \mathrm{AZ}$ - Sint Jan , Brugge, Belgium. ${ }^{126}$ Hochgebirgsklinik Davos, Davos Wolfgang, Switzerland. ${ }^{127}$ Zuyderland, Heerlen, the Netherlands. ${ }^{128}$ Moters ir vaiko klinikos (Woman and Child Clinics), Šiauliai, Lithuania. ${ }^{129}$ Mylan, Vienna, Austria. ${ }^{130}$ Queen Elizabeth Hospital, Glasgow, UK. ${ }^{131}$ Midland General Hospital , Portloise , Ireland. ${ }^{132}$ Praxis, Gensingen, Germany. ${ }^{133}$ Medway Hospital NHS Trust, Gillingham, UK. ${ }^{134}$ Praxis, Heilbronn, Germany. ${ }^{135}$ Sherwood Forest 
NHS Trust, Sutton-in-Ashfield, UK. ${ }^{136}$ Praxis, Berlin, Germany. ${ }^{137}$ University Clinic of Respiratory and Allergic Diseases , Golnik, Slovenia. ${ }^{138}$ Noordwest Ziekenhuisgroep, Almaar, Netherlands. ${ }^{139}$ Saint-Joseph Hospital, Saint-Vith, Belgium. ${ }^{140}$ Odessa National Medical University, Odessa, Ukraine. ${ }^{141} \mathrm{AZ}$ Sint-Jan, Brugge, Belgium. ${ }^{142}$ ALK Benelux, Amere, Netherlands. ${ }^{143}$ AZ Diest, Diest, Belgium. ${ }^{144}$ National Academy of Medicine of Ukraine "Institute of Otorhinolaryngology named of Prof. Kolomiychenko, Kiev, Ukraine. ${ }^{145}$ Frimley Park Hospital, Camberley, UK. ${ }^{146}$ Leeds General Infirmary, Leeds, UK. ${ }^{147}$ University Clinical Center, Maribor, Slovenia.

\section{Authorship contribution}

$\mathrm{PWH}, \mathrm{SFS}, \mathrm{JB}, \mathrm{BP}$ conceived and designed the manuscript; PWH, SFS, JB, BP wrote the manuscript; All authors critically reviewed and revised the manuscript. All authors read and approved the final manuscript.

\section{Conflict of interest}

The authors do not report any conflict of interest related to this manuscript..

\section{References}

1. Zuberbier T, Lötvall J, Simoens S Subramanian SV, Church MK. Economic burden of inadequate management of allergic diseases in the European Union: a GA(2) LEN review. Allergy. 2014;69(10):1275-9.

2. Bousquet J, Khaltaev N. Global surveillance, prevention and control of chronic respiratory diseases: a comprehensive approach [Internet]. Global Alliance against Chronic Respiratory Diseases. World Health Organization. 2007 [cited 2019 Jan 6]. 148 p. Available from: https://www.who.int/gard/ publications/GARD_Manual/en/

3. Blomme K, Tomassen P, Lapeere H, Huvenne W, Bonny M, Acke F, et al. Prevalence of allergic sensitization versus allergic rhinitis symptoms in an unselected population. Int Arch Allergy Immunol. 2013;160(2):200-7.

4. Bousquet PJ, Demoly P, Devillier P, Mesbah K, Bousquet J. Impact of allergic rhinitis symptoms on quality of life in primary care. Int Arch Allergy Immunol. 2013;160(4):393400.

5. Vandenplas O, Vinnikov D, Blanc PD, Agache I, Bachert C, Bewick M, et al. Impact of Rhinitis on Work Productivity: A Systematic Review. J Allergy Clin Immunol Pract. 2018;6(4):1274-1286.e9.

6. Bousquet J, Van Cauwenberge P, Khaltaev N, Aria Workshop Group, World Health Organization. Allergic rhinitis and its impact on asthma. J Allergy Clin Immunol. 2001;108(5 Suppl):S147-334.

7. Bousquet J, Khaltaev N, Cruz AA, Denburg J, Fokkens WJ, Togias A, et al. Allergic Rhinitis and its Impact on Asthma (ARIA) 2008 update (in collaboration with the World Health Organization, GA(2)LEN and AllerGen). Allergy. 2008;63 Suppl 86:8-160.

8. Brozek JL, Bousquet J, Baena-Cagnani CE, Bonini S, Canonica GW, Casale TB, et al. Allergic Rhinitis and its Impact on Asthma (ARIA) guidelines: 2010 revision. J Allergy Clin Immunol. 2010;126(3):466-76.

9. Bousquet J, Schunemann HJ, Fonseca J, Samolinski B, Bachert C, Canonica GW, et al. MACVIA-ARIA Sentinel Network for allergic rhinitis (MASK-rhinitis): the new generation guideline implementation. Allergy. 2015;70(11):1372-92
10. Bousquet J, Hellings PW, Agache I Bedbrook A, Bachert C, Bergmann KC, et al ARIA 2016: Care pathways implementing emerging technologies for predictive medicine in rhinitis and asthma across the life cycle. Clin Transl Allergy. 2016;6(1):47.

11. Bousquet J, Hellings PW, Agache I, Amat F, Annesi-Maesano I, Ansotegui IJ, et al. Allergic Rhinitis and its Impact on Asthma (ARIA) Phase 4 (2018): Change management in allergic rhinitis and asthma multimorbidity using mobile technology. J Allergy Clin Immunol. 2018; 143(3):864-879.

12. Hellings PW, Fokkens WJ, Akdis C, Bachert C, Cingi C, Dietz de Loos D, et al. Uncontrolled allergic rhinitis and chronic rhinosinusitis: where do we stand today? Allergy 2013;68(1):1-7.

13. Smith P, Price D, Harvey R, Carney AS, Kritikos V, Bosnic-Anticevich SZ, et al. Medication-related costs of rhinitis in Australia: a NostraData cross-sectional study of pharmacy purchases. J Asthma Allergy. 2017;10:153-61

14. Bousquet J, Arnavielhe S, Bedbrook A, Bewick M, Laune D, Mathieu-Dupas E, et al. MASK 2017: ARIA digitally-enabled, integrated, person-centred care for rhinitis and asthma multimorbidity using real-worldevidence. Clin Transl Allergy. 2018;8:45.

15. Bousquet J, Devillier P, Arnavielhe S, Bedbrook A, Alexis-Alexandre G, van Eerd $M$, et al. Treatment of allergic rhinitis using mobile technology with real-world data: The MASK observational pilot study. Allergy. 2018;73(9):1763-74

16. Menditto E, Costa E, Midão L, BosnicAnticevich S, Novellino E, Bialek S, et al. Adherence to treatment in allergic rhinitis using mobile technology. the mask study. Clin Exp Allergy. 2019 Apr;49(4):442-460.

17. Cingi C, Gevaert P, Mösges R, Rondon C, Hox V, Rudenko M, et al. Multimorbidities of allergic rhinitis in adults: European Academy of Allergy and Clinical Immunology Task Force Report. Clin Transl Allergy. 2017;7:17

18. Siroux V, Ballardini N, Soler M, Lupinek C, Boudier A, Pin I, et al. The asthma-rhinitis multimorbidity is associated with IgE polysensitization in adolescents and adults.
Allergy. 2018;73(7):1447-58.

19. Amaral R, Bousquet J, Pereira AM, Araújo LM, Sá-Sousa A, Jacinto $T$, et al. Disentangling the heterogeneity of allergic respiratory diseases by latent class analysis reveals novel phenotypes. Allergy. 2019 Apr;74(4):698-708.

20. Amaral R, Fonseca JA, Jacinto T, Pereira AM, Malinovschi A, Janson C, et al. Having concomitant asthma phenotypes is common and independently relates to poor lung function in NHANES 2007-2012. Clin Transl Allergy. 2018;8:13.

21. Anto JM, Bousquet J, Akdis M, Auffray C, Keil T, Momas I, et al. Mechanisms of the Development of Allergy (MeDALL): Introducing novel concepts in allergy phenotypes. J Allergy Clin Immunol. 2017;139(2):388-99.

22. Pinart M, Benet M, Annesi-Maesano I, von Berg A, Berdel D, Carlsen KCL, et al. Comorbidity of eczema, rhinitis, and asthma in IgE-sensitised and non-IgE-sensitised children in MeDALL: a populationbased cohort study. Lancet Respir Med. 2014;2(2):131-40.

23. Gough $H$, Grabenhenrich $L$, Reich $A$, Eckers $\mathrm{N}$, Nitsche O, Schramm D, et al. Allergic multimorbidity of asthma, rhinitis and eczema over 20 years in the German birth cohort MAS. Pediatr Allergy Immunol Off Publ Eur Soc Pediatr Allergy Immunol. 2015;26(5):431-7.

24. Bousquet J, Devillier P, Anto JM, Bewick M Haahtela T, Arnavielhe S, et al. Daily allergic multimorbidity in rhinitis using mobile technology: A novel concept of the MASK study. Allergy. 2018;73(8):1622-31.

25. Siroux V, Boudier A, Nadif R, Lupinek C, Valenta R, Bousquet J. Association between asthma, rhinitis, and conjunctivitis multimorbidities with molecular lgE sensitization in adults. Allergy. 2019;74(4):824-827.

26. Juhn YJ. Risks for Infection in Patients With Asthma (or Other Atopic Conditions): Is Asthma More Than a Chronic Airway Disease? J Allergy Clin Immunol. 2014;134(2):247-257.e3.

27. Kreiner-Møller E, Chawes BLK, CayeThomasen P, Bønnelykke K, Bisgaard $\mathrm{H}$. Allergic rhinitis is associated with otitis 
media with effusion: a birth cohort study Clin Exp Allergy. 2012:42(11):1615-20.

28. Muñoz-Cano R, Ribó P, Araujo G, Giralt E, Sanchez-Lopez J, Valero A. Severity of allergic rhinitis impacts sleep and anxiety: results from a large Spanish cohort. Clin Transl Allergy. 2018;8:23.

29. Leger D, Bonnefoy B, Pigearias B, de La Giclais B, Chartier A. Poor sleep is highly associated with house dust mite allergic rhinitis in adults and children. Allergy Asthma Clin Immunol. 2017;13:36.

30. Pite H, Pimenta L, Henriques AC, Marques I, Camarinha C, Lourenco AV, et al. Lower airway flow influences peak nasal inspiratory flow in school-aged children. Rhinology. 2018;56(3):288-96.

31. Izquierdo-Dominguez A, Jauregui I, Del Cuvillo A, Montoro J, Davila I, Sastre J, et al. Allergy rhinitis: similarities and differences between children and adults. Rhinology. 2017;55(4):326-31.

32. Lamb CE, Ratner PH, Johnson CE Ambegaonkar AJ, Joshi AV, Day D, et al. Economic impact of workplace productivity losses due to allergic rhinitis compared with select medical conditions in the United States from an employer perspective. Curr Med Res Opin. 2006;22(6):1203-10.

33. Guilemany JM, García-Piñero A, Alobid I, Cardelús S, Centellas S, Bartra J, et al. Persistent allergic rhinitis has a moderate impact on the sense of smell, depending on both nasal congestion and inflammation. The Laryngoscope. 2009;119(2):233-8.

34. Mariño-Sanchez F, Valls-Mateus M, Haag O, Alobid I, Bousquet J, Mullol J. Smell loss is associated with severe and uncontrolled disease in children and adolescents with persistent allergic rhinitis. J Allergy Clin Immunol Pract. 2018;6(5):1752-1755.e3.

35. Audino P, La Grutta S, Cibella F, La Grutta S, Melis MR, Bucchieri S, et al. Rhinitis as a risk factor for depressive mood in pre-adoles cents: a new approach to this relationship. Pediatr Allergy Immunol Off Publ Eur Soc Pediatr Allergy Immunol. 2014;25(4):360-5.

36. Vuurman EFPM, Vuurman LL, Lutgens I, Kremer B. Allergic rhinitis is a risk factor for traffic safety. Allergy. 2014;69(7):906-12.

37. Reiner PB, Kamondi A. Mechanisms of antihistamine-induced sedation in the human brain: $\mathrm{H} 1$ receptor activation reduces a background leakage potassium current. Neuroscience. 1994;59(3):579-88.

38. Bachert C, Bousquet J, Hellings P. Rapid onset of action and reduced nasal hyperreactivity: new targets in allergic rhinitis management. Clin Transl Allergy. 2018;8:25.

39. Scadding G, Bousquet J, Bachert C, Fokkens WJ, Hellings PW, Prokopakis E, et al. Rhinology future trends: 2017 EUFOREA debate on allergic rhinitis. Rhinology. 2019 Feb 1:57(1):49-56

40. Fokkens WJ, Pugin B, Bachert C, Bousquet J, Joos G, Louis R, et al. Rhinology Future Debates 2017 by EUFOREA: Novel treatments and surgical solutions in rhinology. Clin Otolaryngol. 2018:43(6):1429-38.
41. Hellings PW, Pugin B, Mariën G, Bachert C, Breynaert C, Bullens DM, et al. Stepwise approach towards adoption of allergen immunotherapy for allergic rhinitis and asthma patients in daily practice in Belgium: a BelSACI-Abeforcal-EUFOREA statement. Clin Transl Allergy. 2019;9:1.

42. Reitsma S, Subramaniam S, Fokkens WWJ, Wang DY. Recent developments and highlights in rhinitis and allergen immunotherapy. Allergy. 2018;73(12):2306-13.

43. Dhami S, Kakourou A, Asamoah F, Agache I, Lau S, Jutel M, et al. Allergen immunotherapy for allergic asthma: A systematic review and meta-analysis. Allergy. 2017;72(12):1825-48.

44. Roberts G, Pfaar O, Akdis CA, Ansotegui IJ, Durham SR, Wijk RG van, et al. EAACl Guidelines on Allergen Immunotherapy: Allergic rhinoconjunctivitis. Allergy. 2018;73(4):765-98.

45. O'Byrne PM, FitzGerald JM, Bateman ED, Barnes PJ, Zhong N, Keen C, et al. Inhaled Combined Budesonide-Formoterol as Needed in Mild Asthma. N Engl J Med. 2018;378(20):1865-76.

46. Camargos P, Affonso A, Calazans G, Ramalho $L$, Ribeiro $M L$, Jentzsch $N$, et al. On-demand intermittent beclomethasone is effective for mild asthma in Brazil. Clin Transl Allergy. 2018;8:7.

47. Bosnic-Anticevich S, Costa E, Menditto $E_{1}$ Lourenço O, Novellino E, Bialek S, et al. ARIA pharmacy 2018 "Allergic rhinitis care pathways for community pharmacy." Allergy. 2018. doi: 10.1111/all.13701. [Epub ahead of print]

48. Flokstra-de Blok BMJ, Brakel TM, Wubs M Skidmore B, Kocks JWH, Oude Elberink JNG, et al. The feasibility of an allergy management support system (AMSS) for IgEmediated allergy in primary care. Clin Transl Allergy. 2018;8:18.

49. Berwick DM. What "Patient-Centered" Should Mean: Confessions Of An Extremist. Health Aff (Millwood). 2009;28(4):w555-65.

50. Seys SF, Bousquet J, Bachert C, Fokkens WJ, Agache I, Bernal-Sprekelsen M, et al. mySinusitisCoach: patient empowerment in chronic rhinosinusitis using mobile technology. Rhinology. 2018;56(3):209-15.

51. Sleurs K, Seys S, Bousquet J, Fokkens W Gorris S, Pugin B, et al. Mobile health tools for the management of chronic respiratory diseases. Allergy. 2019. doi: 10.1111/ all.13720. [Epub ahead of print]

52. Haahtela T, Tuomisto LE, Pietinalho A, Klaukka T, Erhola M, Kaila M, et al. A 10 year asthma programme in Finland: major change for the better. Thorax. 2006;61 (8):663-70.

53. Haahtela T, Valovirta E, Bousquet J, Mäkelä M. The Finnish Allergy Programme 2008-2018 works. Eur Respir J. 2017;49(6):1700470.

54. Bousquet J, Anto JM, Annesi-Maesano I, Dedeu T, Dupas E, Pépin J-L, et al. POLLAR: Impact of air POLLution on Asthma and Rhinitis; a European Institute of Innovation and Technology Health (EIT Health) project. Clin Transl Allergy. 2018;8(1):36.

55. Hellings PW, Borrelli D, Pietikainen S, Agache I, Akdis C, Bachert C, et al. European Summit on the Prevention and Self-Management of Chronic Respiratory Diseases: report of the European Union Parliament Summit (29 March 2017). Clin Transl Allergy. 2017;7:49.

56. Pfaar O, Alvaro M, Cardona V, Hamelmann E, Mösges R, Kleine-Tebbe J. Clinical trials in allergen immunotherapy: current concepts and future needs. Allergy. 2018;73(9):177583.

57. Price D, Bateman ED, Chisholm A, Papadopoulos NG, Bosnic-Anticevich S, Pizzichini $E$, et al. Complementing the randomized controlled trial evidence base. Evolution not revolution. Ann Am Thorac Soc. 2014;11 Suppl 2:S92-98.

58. Konstantinou GN. Pragmatic trials: how to adjust for the "Hawthorne effect"? Thorax. 2012;67(6):562-562.

59. Roche N, Reddel HK, Agusti A, Bateman ED, Krishnan JA, Martin RJ, et al. Integrating real-life studies in the global therapeutic research framework. Lancet Respir Med. 2013;1(10):e29-30.

60. Klimek L, Brehler R, Bergmann K-C. Allergenspecific immunotherapy with storage mites. Allergo J Int. 2018;27(1):15-9.

61. Brehler R, Klimek L, Vogelberg C, Werfel T, Pfaar O, Hamelmann E. Evidence vs. efficacy in allergen-specific immunotherapy: Considerations using the example of tradable products in Germany. Allergo J Int. 2016;25(2):38-43.

62. Klimek L, Fox G-C, Thum-Oltmer S. SCIT with a high-dose house dust mite allergoid is well tolerated: safety data from pooled clinical trials and more than 10 years of daily practice analyzed in different subgroups. Allergo J Int. 2018;27(5):131-9.

63. Reinhold T, Brüggenjürgen B. Costeffectiveness of grass pollen SCIT compared with SLIT and symptomatic treatment. Allergo J Int. 2017;26(1):7-15.

64. Asaria M, Dhami S, Ree R van, Wijk RG van, Muraro A, Roberts $G$, et al. Health economic analysis of allergen immunotherapy for the management of allergic rhinitis, asthma, food allergy and venom allergy: A systematic overview. Allergy. 2018;73(2):269-83.

65. Wahn U, Bachert $C$, Heinrich J, Richter $H_{\text {, }}$ Zielen S. Real-world benefits of allergen immunotherapy for birch pollen-associated allergic rhinitis and asthma. Allergy. 2019;74(3):594-604.

66. Zielen S, Devillier P, Heinrich J, Richter H, Wahn U. Sublingual immunotherapy provides long-term relief in allergic rhinitis and reduces the risk of asthma: A retrospective, real-world database analysis. Allergy. 2018;73(1):165-77.

67. Klimek $L$, Bergmann K-C, Biedermann $T$, Bousquet J, Hellings P, Jung K, et al. Visual analogue scales (VAS): Measuring instruments for the documentation of symptoms and therapy monitoring in cases of allergic 
rhinitis in everyday health care: Position Paper of the German Society of Allergology (AeDA) and the German Society of Allergy and Clinical Immunology (DGAKI), ENT Section, in collaboration with the working group on Clinical Immunology, Allergology and Environmental Medicine of the German Society of Otorhinolaryngology, Head and Neck Surgery (DGHNOKHC). Allergo J Int. 2017;26(1):16-24

68. Del Cuvillo A, Santos V, Montoro J, Bartra J, Davila I, Ferrer M, et al. Allergic rhinitis severity can be assessed using a visual analogue scale in mild, moderate and severe. Rhinology. 2017;55(1):34-8.

69. Bousquet J, Bewick M, Arnavielhe S, Mathieu-Dupas E, Murray R, Bedbrook A et al. Work productivity in rhinitis using cell phones: The MASK pilot study. Allergy. 2017;72(10):1475-84

70. Bousquet J, Murray R, Price D, Somekh D, Münter L, Phillips J, et al. The allergic allergist behaves like a patient. Ann Allergy Asthma Immunol Off Publ Am Coll Allergy Asthma Immunol. 2018;121(6):741-2.
Prof. Dr. Peter W. Hellings

Clinical Division of Otorhinolaryngo-

logy, Head and Neck Surgery

University Hospitals Leuven

KU Leuven

Kapucijnenvoer 33

3000 Leuven

Belgium

Tel: +3216332338

Fax: +32 16346035

E-mail: Peter.hellings@kuleuven.be 